\title{
Evaluación de los resultados de la cirugía reconstructiva del esqueleto mitroaórtico en la endocarditis infecciosa activa
}

\author{
Lucio Sartor*, Ulises Ramírez Valdiris, José Antonio Blázquez, \\ Omar Al Razzo y José María Mesa García
}

Servicio de Cirugía Cardíaca, Hospital Universitario La Paz, Madrid, España

Recibido el 24 de enero de 2016; aceptado el 10 de julio de 2016

Disponible en Internet el 23 de noviembre de 2016

\author{
PALABRAS CLAVE \\ Endocarditis; \\ Endocarditis \\ protésica; \\ Fuga perivalvular; \\ Infección; \\ Cirugía cardiaca
}

\begin{abstract}
Resumen
Introducción: La cirugía en la endocarditis infecciosa activa con múltiples abscesos y destrucción del cuerpo fibroso intervalvular, representa un procedimiento de alta exigencia técnica y de difícil manejo postoperatorio. Se presenta la experiencia con una técnica original de resección radical y reconstrucción posterior con pericardio bovino.

Material y métodos: En los últimos ocho años en nuestro centro se intervinieron 29 pacientes con endocarditis infecciosa activa y abscesos paravalvulares que destruían la unión mitro-aórtica (20 sobre prótesis y 9 sobre válvula nativa): 13 de ellos llegaron al quirófano en situación de sepsis grave. El EuroScore I medio de la serie fue $36 \pm 22,7 \%$. Para tratarlos se realizó una resección amplia del tejido infectado y posterior reconstrucción del cuerpo fibroso con pericardio bovino fijado en glutaraldehído, mediante una técnica original.

Resultados: La mortalidad hospitalaria de la serie fue del 20,7\%. El seguimiento medio fue de $34,2 \pm 28$ meses con un máximo de 8 años. Dos pacientes requirieron reintervención a causa de fugas periprotésicas aórticas. Se registró una sola recidiva del proceso infeccioso. Dos pacientes fallecieron durante el seguimiento posterior al alta, por causas no cardíacas.

Conclusiones: La resección amplia de la unión mitro-aórtica y la posterior reconstrucción con pericardio bovino, en algunos casos puede ser la única opción quirúrgica para salvar la vida de un paciente. Los autores consideran que la técnica presentada es un procedimiento reproducible, con morbilidad y mortalidad aceptables, y con el que se minimizaría el riesgo de recidivas futuras.

(C) 2016 Sociedad Colombiana de Cardiología y Cirugía Cardiovascular. Publicado por Elsevier España, S.L.U. Este es un artículo Open Access bajo la licencia CC BY-NC-ND (http:// creativecommons.org/licenses/by-nc-nd/4.0/).
\end{abstract}

\footnotetext{
* Autor para correspondencia.

Correo electrónico: luciosartor@gmail.com (L. Sartor).
} 


\section{KEYWORDS}

Endocarditis;

Prosthetic

endocarditis;

Perivalvular leak;

Infection;

Cardiac surgery

\section{Evaluation of the results of surgical reconstruction of the mitroaortic skeleton in active infective endocarditis}

\begin{abstract}
Introduction: Surgical intervention of active infective endocarditis with multiple abscesses and destruction of the intervalvular fibrous body represents a technically demanding procedure with a difficult postoperative management. The experience is presented with an original technique for radical resection and subsequent reconstruction using bovine pericardium.

Material and methods: During the last eight years in our centre 29 patients with active infective endocarditis and paravalvular abscesses that destroyed the microaortic union underwent surgery (20 over prosthesis and 9 over native valve): 13 of them reached the operating room with severe sepsis. Average EuroScore । of the series was $36 \pm 22.7 \%$. Treatment consisted of a broad resection of infected tissue and subsequent reconstruction of the fibrous body with glutaraldehyde fixed bovine pericardium, by means of an original technique.

Results: Hospital mortality of the series was $20.7 \%$. Average follow-up was $34.2 \pm 28$ months, with a maximum of 8 years. Two patients required a reoperation due to periprosthetic aortic valve leaks. Only one relapse of the infectious process was registered. Two patients passed away during follow-up after discharge, for non-cardiac reasons.

Conclusion: Broad resection of the mitroaortic union and subsequent reconstruction with bovine pericardium can in some cases be the only surgical option to save a patient's life. The authors consider that the presented technique is a repeatable procedure, with acceptable morbidity and mortality, that would minimise the risk of future recurrence.

(c) 2016 Sociedad Colombiana de Cardiología y Cirugía Cardiovascular. Published by Elsevier España, S.L.U. This is an open access article under the CC BY-NC-ND license (http:// creativecommons.org/licenses/by-nc-nd/4.0/).
\end{abstract}

\section{Introducción}

La zona de continuidad fibrosa entre las válvulas mitral y aórtica se describe como el esqueleto mitro-aórtico. Esta estructura está implicada en la integridad anatómica y funcional de ambas válvulas ${ }^{1}$. La endocarditis infecciosa con afectación de las válvulas aórtica y mitral, que se acompaña de complicaciones perianulares infecciosas, incluyendo abscesos, pseudoaneurismas y fístulas, denota una fase de enfermedad avanzada, con claros indicadores de mal pronóstico ${ }^{2}$. Por este motivo, estos pacientes son considerados por la mayoría de los cirujanos, como los casos más difíciles de abordar ${ }^{3}$. En la actualidad, la estrategia quirúrgica aceptada para el tratamiento de la endocarditis aórtica acompañada de abscesos, consiste en desbridamiento radical del tejido infectado y su reconstrucción posterior ${ }^{4,5}$. No obstante, la operación de la endocarditis en fase avanzada sigue siendo un desafío quirúrgico, con tasas de mortalidad que aún hoy permanecen elevadas $2,6,7$.

A continuación se describe nuestra experiencia acumulada en los últimos ocho años, con una técnica original de reconstrucción de la unión mitro-aórtica para tratar los casos de endocarditis con afectación paravalvular severa.

\section{Material y métodos}

\section{Población}

Estudio restrospectivo, con datos obtenidos de un solo centro, desde enero de 2006 hasta septiembre de 2014, período
Tabla 1 Características preoperatorias

\begin{tabular}{lcc}
\hline Variable & Valor & $\%$ \\
\hline Edad & 64 & \\
Sexo & & \\
$\quad$ Hombres & 23 & 79,3 \\
$\quad$ Mujeres & 6 & 20,7 \\
Clase NHYA & & \\
$\quad$ III & 14 & 48,3 \\
IV & 15 & 51,7 \\
HTA & 21 & 72,4 \\
DM & 9 & 31 \\
IRC & 10 & 34,5 \\
Embolia SNC & 2 & 6,9 \\
Shock séptico & 13 & 44,8 \\
Fracción de eyección < 40\% & 10 & 34,5 \\
\hline
\end{tabular}

HTA: hipertensión arterial. DM: diabetes mellitus IRC: insuficiencia renal crónica. SNC: sistema nervioso central.

en el que se intervinieron 29 pacientes con diagnóstico de endocarditis infecciosa con afectación de la continuidad mitro-aórtica, mediante una técnica original de reconstrucción del esqueleto fibroso.

Las características preoperatorias de los pacientes intervenidos se reflejan en las tablas 1 y 2 .

El 58,6\% de los pacientes se intervino de forma urgente y el $41,4 \%$ de manera programada.

El EuroScore I logístico medio de estos pacientes fue de $36 \pm 22,7 \%$ con un rango entre 4 y $81 \%$. En todos los casos se hicieron ecocardiogramas transtorácicos y transesofágicos 
Tabla 2 Características preoperatorias

\begin{tabular}{lcr}
\hline Variable & Valor & $\%$ \\
\hline Microorganismos & & \\
Staphylococcus epidermidis & 11 & 37,9 \\
Staphylococcus aureus & 6 & 20,7 \\
Enterococcus feacalis & 3 & 10,3 \\
Gamella haemolisans & 2 & 6,9 \\
Esteril & 2 & 6,9 \\
Otros & 8 & 27,6 \\
Prótesis previa & & \\
Prótesis mecánica & 11 & 38 \\
Prótesis biológica & 9 & 31 \\
Nativa & 9 & 31 \\
\hline
\end{tabular}

preoperatorios y se extrajeron hemocultivos y cultivos del tejido infectado obtenidos durante la intervención quirúrgica.

Un 51,7\% llegó al quirófano con tratamiento antibiótico orientado con antibiograma derivado de un hemocultivo positivo previo, en tanto que el $48,3 \%$ debieron ser tratados con antibioticoterapia de amplio espectro por ausencia de identificación del microorganismo implicado en el momento de la intervención. El tiempo de tratamiento antibiótico previo a la intervención, varió de 1 a 30 días, con una media de $8,2 \pm 8$ días.

\section{Técnica quirúrgica}

Se trata de una técnica original, que surge como modificación de la técnica descrita inicialmente por T. David ${ }^{8}$. Toda la serie fue intervenida por un mismo cirujano. Todos los procedimientos se realizaron con soporte de circulación extracorpórea convencional, con cardioplejía hemática por vía anterógrada y retrógrada. Se accedió a través de esternotomía media. La técnica inicia con una aortotomía en $\mathrm{S}$ itálica con progresión hacia la continuidad mitro-aórtica, a nivel de la comisura entre el velo no coronario y el coronario izquierdo, tal cual se describe en la técnica original.

Se extirpa la válvula aórtica nativa o la prótesis aórtica. Se practica un desbridamiento amplio de todas las zonas infectadas entre ambos trígonos, resecando la válvula mitral a través de la misma aortotomía, preservando el aparato subvalvular posterior, siempre que no esté afectado por el proceso infeccioso. A diferencia de la técnica original se procede al implante de la prótesis mitral antes de la reconstrucción de la continuidad mitro-aórtica. Se implanta en sus dos tercios posteriores a través de la aortotomía, con puntos de Ti-Cron ${ }^{\circledR}$ de 2-0 apoyados en teflón de $7 \times 3 \mathrm{~mm}$, hasta ambos trígonos fibrosos (fig. 1). En esta técnica empleada, a diferencia de los dos parches descritos por David, se usa un solo parche de pericardio bovino fijado en glutaraldehído (Peri-Guard $^{\circledR}$ ) en forma rectangular (de unos $35 \times 70 \mathrm{~mm}$, dependiendo de la distancia intertrigonal). Este parche se une al tercio restante de la prótesis mitral mediante una sutura continua de Prolene ${ }^{\circledR}$ de 3-0 (fig. 2). Quedan así conformadas dos hojas, una para cerrar el techo de la aurícula izquierda y otra para ampliar y cerrar la raíz aórtica. Se recorta la hoja externa del parche dándole forma triangular

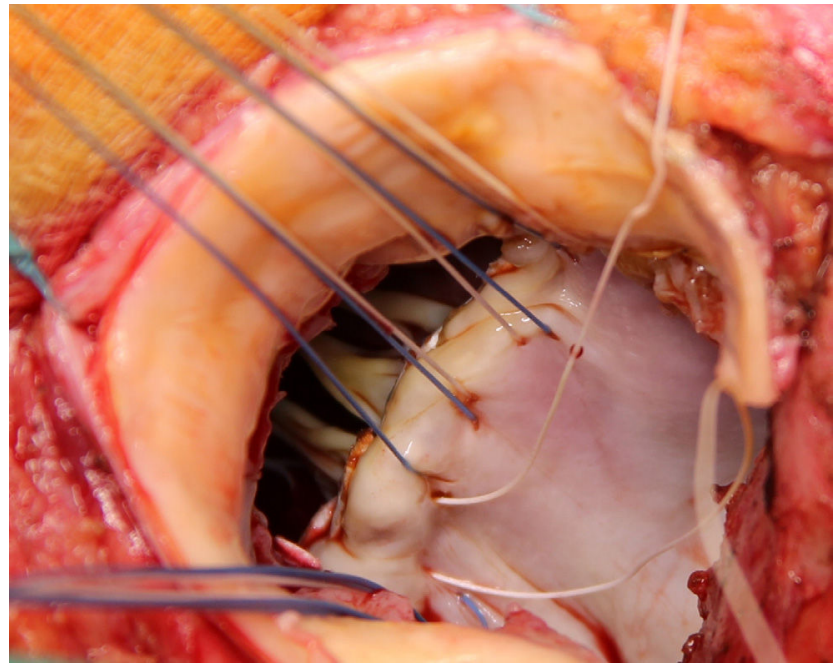

Figura 1 Implante de puntos mitrales realizado a través de la aortotomía ampliada.

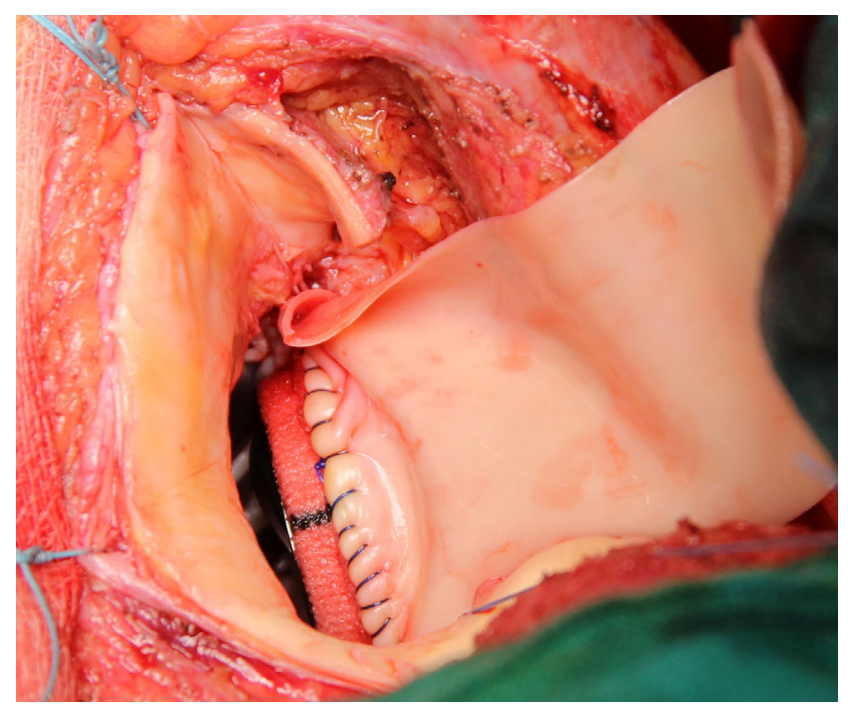

Figura 2 Prótesis mitral implantada en sus dos tercios posteriores. Sutura del parche de pericardio bovino a la prótesis mitral.

y se cierra el techo de la aurícula izquierda con éste. Se implantan puntos de Ti-Cron ${ }^{\circledR}$ de 2-0 apoyados en teflón de $7 \times 3 \mathrm{~mm}$, primero en la otra hoja del parche de pericardio destinado a ampliar y cerrar la raíz aórtica. El resto de los puntos se ubican en el anillo aórtico, procurando apoyarlos en tejido sano (fig. 3). Se pasan los puntos por el anillo de la prótesis aórtica dejando ésta en posición supraanular (fig. 4). Una vez implantada la prótesis aórtica, se reconstruye la raíz aórtica con la hoja del parche de pericardio bovino que previamente se suturó a la prótesis aórtica.

En 19 pacientes se implantaron prótesis mecánicas y en otros 10, prótesis biológicas. Tres casos requirieron sustitución de la aorta ascendente concomitante, uno reconstrucción compleja del tabique interventricular con parche de pericardio, uno reconstrucción de la aurícula derecha por la presencia de una fístula y otro cirugía de revascularización coronaria concomitante. 


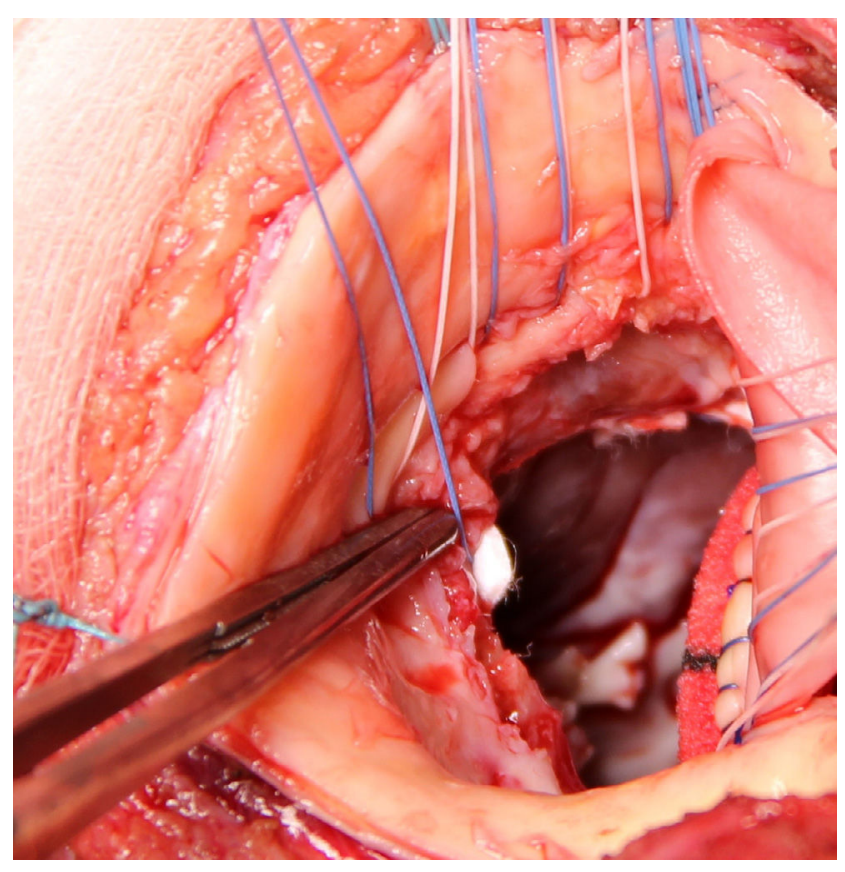

Figura 3 Implante de puntos aórticos por debajo del anillo. Implante de puntos aórticos desde fuera del parche de pericardio bovino.

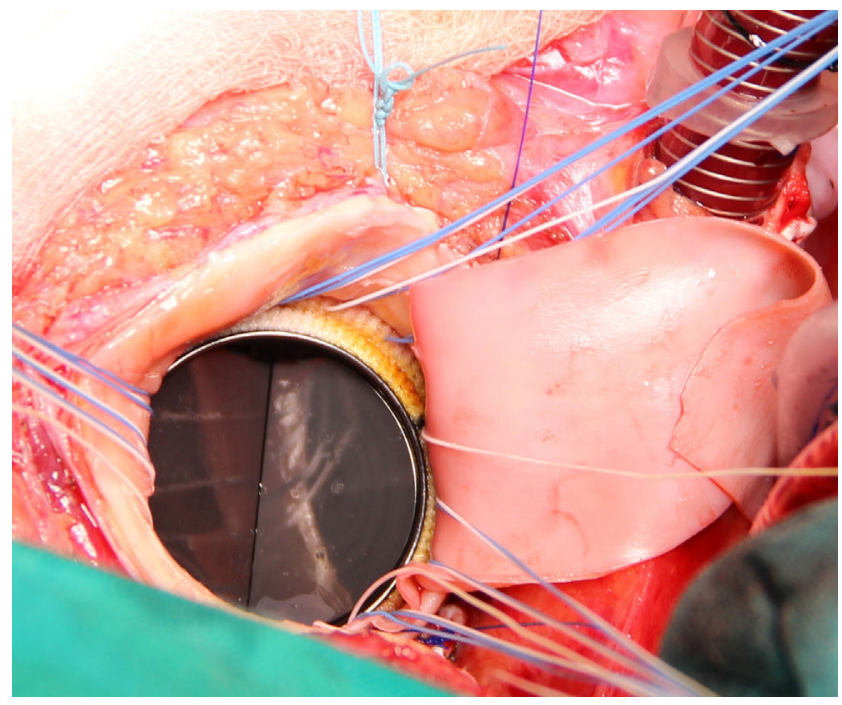

Figura 4 Implante de la prótesis aórtica en posición supraanular.

\section{Seguimiento}

Se estableció un protocolo que consistió en realizar un ecocardiograma transtorácico antes del alta hospitalaria, uno a los 6 meses y otro antes de cada revisión anual. La media de seguimiento fue de $34,2 \pm 28$ meses con un rango de 4 días a 8 años. El seguimiento a un año pudo hacerse en 21 pacientes, mientras que el de 3 años en 14 pacientes. No se perdieron pacientes durante el seguimiento. Se tomó como fecha de inicio del seguimiento aquella de la intervención quirúrgica.

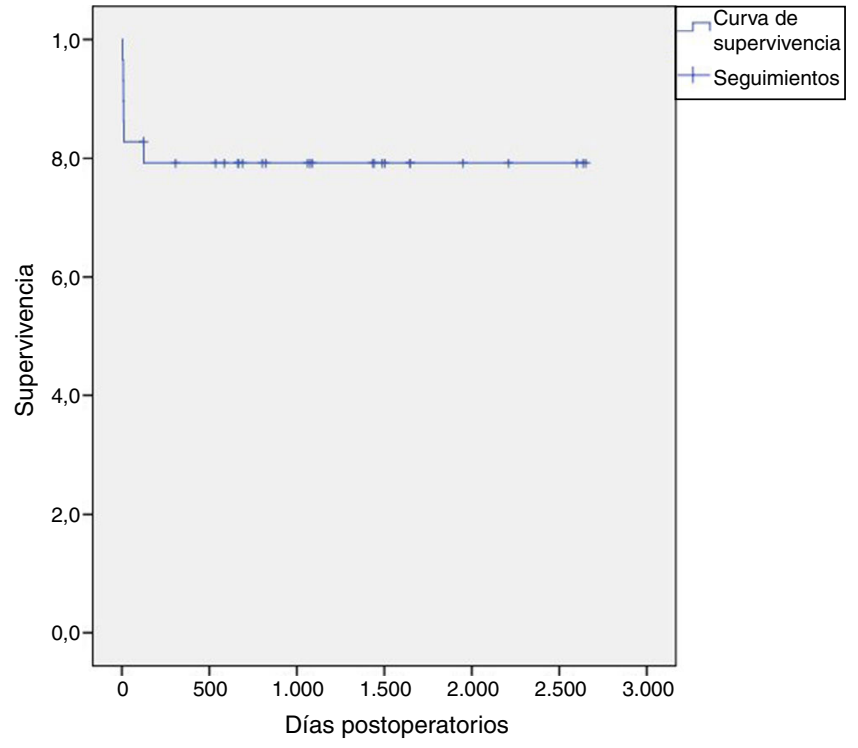

Figura 5 Curva de supervivencia actuarial.

Tabla 3 Complicaciones postoperatorias

\begin{tabular}{llr}
\hline Variable & Valor & \multicolumn{1}{c}{$\%$} \\
\hline Fugas periprotésicas & 2 & 6,9 \\
Reintervención por sangrado & 4 & 13,8 \\
BAV con implante de marcapasos & 6 & 20,7 \\
IRA con necesidad de hemofiltro/diálisis & 6 & 20,7 \\
Neumonía & 5 & 17,2 \\
ILQ & 1 & 3,4 \\
Mediastinitis & 1 & 3,4 \\
Neurológicas & 2 & 6,9 \\
\hline
\end{tabular}

BAV: bloqueo auriculoventricular IRA: insuficiencia renal aguda ILQ: infección del lecho quirúrgico.

\section{Resultados}

Se registró una mortalidad hospitalaria del 20,7\% (6 pacientes); todos fallecidos en el contexto de sepsis no controlada con falla multiorgánica añadida dentro del mismo ingreso hospitalario (fig. 5). No se registró mortalidad en el seguimiento extrahospitalario. La media de los tiempos de circulación extracorpórea y de isquemia fue de $232 \pm 62$ y $195 \pm 48$ minutos respectivamente. Dos pacientes requirieron reintervención por fuga periprotésica aórtica; uno al mes y el otro a los 4 meses de la intervención (tabla 3 ).

La estancia media postoperatoria en la unidad de cuidados intensivos fue de 17,1 $\pm 29,2$ días (mediana: 10 días, rango: 5-125 días), en tanto que la media de estancia hospitalaria fue de 52,1 $\pm 36,2$ días (mediana: 36,5 rango: 12-124 días).

En las ecocardiografías practicadas a los pacientes en el postoperatorio se observó un funcionamiento correcto de las prótesis mitral y aórtica, sin evidencia de fugas ni fístulas residuales, a excepción de los dos pacientes comentados.

Durante el seguimiento, fallecieron dos pacientes, uno al año de ser intervenido, a causa de problemas respiratorios, y otro a los 3 años a raíz de una hemorragia subaracnoidea. 
De 29 pacientes, 18 permanecen en clase funcional I de la NYHA y 3 en clase $॥$ de la NYHA; se registraron 8 éxitus.

\section{Discusión}

En general, la estrategia quirúrgica aceptada para el tratamiento de la endocarditis aórtica y mitral acompañada de abscesos, consiste en el desbridamiento radical del tejido infectado, a fin de obtener un margen de tejido sano y con ello minimizar el riesgo $0^{4,5}$. A pesar de los avances en el diagnóstico y tratamiento de la endocarditis infecciosa, ésta sigue siendo una entidad con una mortalidad muy elevada, con cifras que rondan el $25 \%^{2,6,7}$. En nuestra serie se registra una mortalidad hospitalaria del 20,7\%. Se destaca que todos estos casos corresponden a pacientes con un perfil preoperatorio de riesgo elevado, en situación de sepsis preoperatoria. El 66,7\% de los fallecidos eran portadores de prótesis intracardiacas previas, y el $83 \%$ de estos se intervinieron de forma urgente.

La mayoría de las series coinciden en que el patógeno responsable más común de las endocarditis con afectación de la continuidad mitro-aórtica es el Staphylococcus aureus (entre un $23 \%$ y $31 \%)^{9,10}$. En esta serie el microorganismo más frecuente fue el Staphylococcus epidermidis (37,9\%), seguido por el Staphylococcus aureus $(20,7 \%)$ y el Enterococcus feacalis $(10,3 \%)$. Esto probablemente se deba a cambios en los microorganismos causales en los últimos años, algo que hace de la endocarditis un proceso a veces más lento pero más destructivo, causante de gran deterioro tisular y difícil de erradicar con tratamiento médico o quirúrgico. La idea de que los microorganismos menos virulentos, como el Streptococcus viridans, siempre responden a los antibióticos, es errónea ya que estas bacterias pueden causar un amplio daño tisular ${ }^{3}$. Así es que en esta serie se registró un caso con dicho patógeno, que ocasionó una endocarditis con amplia destrucción perivalvular, a pesar del tratamiento antibiótico dirigido. A falta de ampliar esta serie, el equipo de investigación no halló diferencias en cuanto a la evolución y el pronóstico en lo que atañe a microorganismos causales de la endocarditis.

El 13,8\% de los pacientes intervenidos requirió revisión en quirófano por sangrado postoperatorio no controlado, aunque ninguno presentó punto sangrante concreto, manifestándose como sangrado en sábana. Tres de los cuatro casos reintervenidos por sangrado se registraron en el primer tercio de la serie, hecho que demuestra la importancia de la curva de aprendizaje en este tipo de procedimientos.

Uno de los pacientes que necesitó reintervención por fuga periaórtica presentaba absceso en la raíz aórtica a nivel de la comisura entre los velos coronario izquierdo y derecho; inicialmente, fue tratado con un parche de pericardio bovino a fin de aislar éste de la circulación. Las fugas se detectaron en el postoperatorio temprano y al mes de la intervención se procedió a la reintervención, tiempo en el que se observó un desprendimiento completo del parche de pericardio. A partir de este momento se sustituyó la técnica clásica de aislamiento con parche de pericardio bovino por puntos apoyados en teflón, fijando la prótesis por debajo del anillo. El segundo caso de fuga periprotésica aórtica correspondió a un paciente que desarrolló recidiva de la endocarditis con desprendimiento parcial de la prótesis aórtica de la inserción del velo coronario derecho. Se destaca que ninguna de las dos fugas periprotésicas comprometían al parche que reemplaza la continuidad mitroaórtica. Se cree que este último hecho se debe a las modificaciones que se han introducido en la técnica, puesto que posiblemente la sutura continua del parche de pericardio al tercio anterior de la prótesis mitral, proporciona un cierre más hermético que aquel que brinda la sutura con puntos sueltos propuestos por la técnica original.

Dada la complejidad del procedimiento, con un desbridamiento amplio del tejido infectado, muchas veces en inmediata vecindad al sistema de conducción, resulta sorprendente la tasa baja de implante de marcapasos definitivo $(20,7 \%)$, datos que coinciden con otras series publicadas (entre el 18 y $24 \%)^{11,12}$.

De otro lado, el ecocardiograma transesofágico es una prueba imprescindible para el diagnóstico temprano y la monitorización de este tipo de intervenciones ${ }^{13,14}$. En esta serie, de 29 pacientes intervenidos, 7 (24,1\%) no tenían diagnóstico preoperatorio de afectación de la continuidad mitro-aórtica. Esto último denota la dificultad diagnóstica asociada a esta entidad, en especial cuando el paciente es portador de prótesis mecánica, dada la distorsión ecocardiográfica que éstas ocasionan.

Los tiempos medios elevados de estancia en la unidad de cuidados intensivos indican el manejo postoperatorio complejo que requieren este tipo de pacientes. Por otro lado, los tiempos de estancia en planta de hospitalización para cuidados no críticos son prolongados, situación que se fundamenta en que todos los pacientes de la serie cumplieron un mínimo de 6 semanas de tratamiento antibiótico intravenoso antes de recibir el alta hospitalaria.

Clásicamente, la cirugía de estos casos ha consistido en la exéresis de la válvula o prótesis afectada con desbridamiento y limpieza de los abscesos. En grandes defectos, en general se busca el aislamiento de estos, de la circulación mediante el uso de parches o bien con cierre directo; incluso algunos autores han propuesto rellenarlos con pegamentos biológicos ${ }^{15}$, mientras otros platean el cambio en bloque de ambas prótesis junto con la continuidad mitro-aórtica, mediante un implante compuesto elaborado en forma artesanal fuera del campo quirúrgico ${ }^{16,17}$. Consideramos que con la técnica expuesta se obtiene mejor adaptación de ambas prótesis con el resto de estructuras cardíacas. Esta adaptación se consigue de forma más sencilla y reproducible cuando se implanta la prótesis mitral antes de la reconstrucción mitro-aórtica. En este último paso, creemos que la técnica propuesta ofrece ventajas sobre la técnica clásica.

Cuando se encuentra afectada la continuidad mitroaórtica debería evitarse la política de "ahorro", puesto que el riesgo de fugas y recidivas es muy elevado en estos casos. Cabe reseñar que la tasa de recidiva de la infección aun empleando las técnicas más radicales, es del $7 \%{ }^{18}$, hasta un $30 \%$ de los pacientes llega a necesitar reintervención a los 10 años ${ }^{19}$. Un buen dato para destacar en esta serie es que solo un paciente requirió reintervención por reinfección, lo cual se presume, puede fundamentarse en la agresividad de la técnica empleada.

En esta práctica existió una tendencia a utilizar prótesis mecánicas sobre las biológicas a pesar de que 8 de estos pacientes tenían más de 65 años. Esto último se explica por 
la complejidad de este tipo de procedimiento y la dificultad de una posible reintervención por deterioro estructural.

Está demostrado que un tratamiento quirúrgico agresivo acompañado de una terapia antibiótica correcta, pueden disminuir la mortalidad en pacientes con endocarditis infecciosa activos de alto riesgo ${ }^{20}$. Por este motivo, además de la estrategia quirúrgica adecuada, es primordial la identificación de los microorganismos responsables y el inicio temprano de un tratamiento antibiótico debidamente dirigido.

Se considera que los buenos resultados obtenidos en esta serie obedecen principalmente a la actitud radical que se adoptó con este tipo de pacientes, minimizándose de esta forma el riesgo de reinfección, dehiscencia y sepsis por persistencia del tejido infectado.

\section{Conclusiones}

La endocarditis infecciosa con afectación de la continuidad mitro-aórtica denota una fase avanzada de este proceso infeccioso, de ahí que se considere que el mejor enfoque que se le puede dar a estos pacientes es el diagnóstico precoz y la actuación inmediata. Una vez existe afectación de la continuidad mitro-aórtica, es preciso eliminar todo el tejido infectado, sin que la magnitud de la reconstrucción interfiera en la decisión.

\section{Responsabilidades éticas}

Protección de personas y animales. Los autores declaran que para esta investigación no se han realizado experimentos en seres humanos ni en animales

Confidencialidad de los datos. Los autores declaran que en este artículo no aparecen datos de pacientes.

Derecho a la privacidad y consentimiento informado. Los autores declaran que en este artículo no aparecen datos de pacientes.

\section{Conflicto de intereses}

Los autores declaran no tener ningún conflicto de intereses.

\section{Bibliografía}

1. Cavalcanti JS, Oliveira E de L, Godoi ET, Santos LP, de Lima e Silva VX, Oliveira M de L. Mesoscopic study of the mitral valve and its fibrous ring. Arq Bras Cardiol. 1997;69:243-6.

2. Mylonakis E, Calderwood SB. Infective endocarditis in adults. N Engl J Med. 2001;345:1318-30.

3. D'Udekem Y, David TE, Feindel CM, Armstrong S, Sun Z. Longterm results of operation for paravalvular abscess. Ann Thorac Surg. 1996;62:48-53.

4. Butchart EG, Gohlke-Bärwolf C, Antunes MJ, Tornos P, De Caterina R, Cormier B, et al. Recommendations for the management of patients after heart valve surgery. Eur Heart J. 2005;246:3-71.
5. Leyh RG, Knobloch K, Hagl C, Ruhparwar A, Fischer S, Kofidis T, et al. Replacement of the aortic root for acute prosthetic valve endocarditis: Prosthetic composite versus aortic allograft root replacement. J Thorac Cardiovasc Surg. 2004;127:1416-20.

6. Greason KL, Thomas M, Steckelberg JM, Daly RC, Schaff HV, Li $Z$, et al. Outcomes of surgery in the treatment of isolated nonnative mitral valve infective endocarditis. J Thorac Cardiovasc Surg. Elsevier;. 2014;147:349-54.

7. Ishikawa S, Kawasaki A, Neya K, Abe K, Suzuki H, Koizumi S, et al. Surgical treatments for infective endocarditis involving valve annulus. Ann Thorac Cardiovasc Surg. 2009;15:378-81.

8. David TE, Kuo J, Armstrong S. Aortic and mitral valve replacement with reconstruction of the intervalvular fibrous body. J Thorac Cardiovasc Surg. 1997;114(5):766-71; discussion 771-2.

9. David TE, Gavra G, Feindel CM, Regesta T, Armstrong S, Maganti $M D$. Surgical treatment of active infective endocarditis: A continued challenge. J Thorac Cardiovasc Surg. 2007;133:144-9.

10. Murdoch DR, Corey GR, Hoen B, Miró JM, Fowler VG, Bayer AS, et al. Clinical presentation, etiology, and outcome of infective endocarditis in the $21^{\text {st }}$. century: the International Collaboration on Endocarditis-Prospective Cohort Study. Arch Intern Med. 2009;169:463-73.

11. David TE, Armstrong S, Maganti M, Ihlberg L. Clinical outcomes of combined aortic root replacement with mitral valve surgery. J Thorac Cardiovasc Surg. 2008;136:82-7.

12. De Oliveira NC, David TE, Armstrong S, Ivanov J. Aortic and mitral valve replacement with reconstruction of the intervalvular fibrous body: an analysis of clinical outcomes. J Thorac Cardiovasc Surg. 2005;129:286-90.

13. Runge MS, Stouffer GA, Sheahan RG, Lerakis S, Robert Taylor W, Lynch $M$, et al. The role of transesophageal echocardiography in the diagnosis and management of patients with aortic perivalvular abscesses. Am J Med Sci. 2001;321:152-5.

14. Mele D, Agricola E, Dal Monte A, Bruno Ancona M, Ferrari R. Evaluation and closure of the periprosthetic leak: Role of realtime three-dimensional transesophageal echocardiography. G Ital Cardiol (Roma). 2012;13:38-46.

15. Farahmand P, Laali M, Renier V, D’Alessandro C, Rama A, Leprince $\mathrm{P}$, et al. Long-term results of aortic root abscess treated with cavity gluing and annular reconstruction by patch. Interact Cardiovasc Thorac Surg. 2012;15:S145.

16. Davierwala PM, Binner C, Subramanian S, Luehr M, Pfannmueller $B$, Etz C, et al. Double valve replacement and reconstruction of the intervalvular fibrous body in patients with active infective endocarditis. Eur J Cardio-thoracic Surg. 2014;45:146-52.

17. Obadia JF, Hénaine R, Bergerot C, Ginon I, Nataf P, Chavanis $\mathrm{N}$, et al. Monobloc aorto-mitral homograft or mechanical valve replacement: a new surgical option for extensive bivalvular endocarditis. J Thorac Cardiovasc Surg [Internet]. Elsevier; 2006 Jan 1 [cited 2014 Oct 21];131(1):243-5. Disponible en: http://www.jtcvsonline.org/article/S0022522305017435/ fulltext

18. David TE. Surgical management of aortic root abscess. J Card Surg. 1997;12:262-9.

19. De Oliveira NC, David TE, Armstrong S, Ivanov J. Aortic and mitral valve replacement with reconstruction of the intervalvular fibrous body: An analysis of clinical outcomes. J Thorac Cardiovasc Surg. 2005;129:286-90.

20. Dohmen PM, Linneweber J, Marz S, Lembcke A, Maneva G, Puhlmann B, et al. Aggressive surgical treatment and appropriate antibiotic therapy can improve outcome of active infective left-side endocarditis. Interact Cardiovasc Thorac Surg. 2009;8:S57-8. 